\title{
Sistema observacional para analizar interacciones didácticas en clases de ciencias en bachillerato
}

\author{
Observational system to analyze didactic interactions in science classes in \\ bachelor
}

\author{
Néstor M. Velarde-Corrales ${ }^{1}$ \\ Centro de Investigación Transdisciplinar en psicología (CITPSI) \\ Aldo Bazán-Ramírez² \\ Universidad Autónoma del Estado de Morelos
}

Recibido: $15-10-19$

Aceptado: $17-12-19$

\begin{abstract}
Resumen
El objetivo del presente trabajo fue, construir y validar un sistema observacional para realizar análisis de interacciones didácticas en dos clases de ciencias en el bachillerato, y describir de manera exploratoria, las relaciones establecidas entre el desempeño del profesor y el desempeño del estudiante. Este estudio se llevó a cabo en tres fases: En la fase 1 se delimitaron las categorías apegadas a un enfoque teórico. En la fase 2, en el software Lynx versión 4.1 se programaron las categorías e indicadores. y se calculó el índice de acuerdo entre observadores. En la fase 3 se probó el sistema de observación, analizando dos vídeo grabaciones de clases de ciencia. De acuerdo con los resultados, este sistema de registro observacional obtuvo buen índice de confiabilidad, mostrando ser útil para analizar las interacciones didácticas. Los datos también mostraron, que los criterios Ilustración y Ajuste al modo linguístico e ilustración, fueron los desempeños más frecuentes en las interacciones observadas. Asimismo, se discute la relevancia del sistema de observación para el apoyo a la práctica docente.
\end{abstract}

Palabras clave: Observación de clases; interacciones; bachillerato; competencias docentes; videograbación.

\footnotetext{
1 Profesor - investigador en la Universidad Autónoma del Estado de Morelos.

E-mail: nestor.velardecorrales@uaem.edu.mx

2 Doctor y maestro en psicología por la Universidad Nacional Autónoma de México y obtuvo el título de psicólogo en la Universidad Nacional Federico Villarreal (Perú). Es profesor - investigador en la Universidad Autónoma del Estado de Morelos, donde también imparte clases y dirige tesis en Licenciatura, Maestría y Doctorado. Es miembro del Sistema Nacional de Investigadores de México (SNI) de manera ininterrumpida desde enero de 2002. Dirigir correspondecia al E-mail: abazan@uaem.mx

(C) Los autores. Este artículo es publicado por la Revista de Investigación en Psicología de la Facultad de Psicología, Universidad Nacional Mayor de San Marcos. Este es un artículo de acceso abierto, distribuido bajo los términos de la licencia Creative Commons Atribucion - No Comercia_Compartir Igual 4.0 Internacional. (http://creativecommons.org/licenses/by-nc-sa/4.0/) que permite el uso no comercial, distribución y reproducción en cualquier medio, siempre que la obra original sea debidamente citada.
} 


\begin{abstract}
The objective of this reasearch was to build and validate an observational system to analysis didactic interactions in two science classes in high school, and to describe in an exploratory way, the relationships established between the performance of the teacher and student performance. This study was carried out in three phases: In phase 1, the categories were delimited according to the theoretical approach. In Phase 2, the categories and indicators were programmed in the Lynx version 4.1 software, and the observer agreement index was calculated. In phase 3 , the observation system was tested, analyzing two video recordings of science classes. According to the results, this observational recording system obtained a good reliability index, proving useful for analyzing teaching interactions. The data also showed that the Illustration and Fit to Linguistic mode and Illustration criteria were the most frequent performances in observed interactions. The relevance of the observation system for supporting teaching practice is also discussed.
\end{abstract}

Keywords: Observation of classes; interactions; high school; teaching skills; video recording.

La observación en clase es una herramienta que puede ser utilizada para la mejora de las prácticas docentes, ya que permite realimentar al docente sobre su desempeño y sus acciones en el aula de acuerdo con los objetivos curriculares, diferentes criterios de enseñanza y de logro, y de acuerdo con las características de sus estudiantes. De esta forma, los sistemas de observación utilizados en entornos escolares permiten al docente realizar un análisis minucioso respecto de sus habilidades en el desarrollo de una clase, así como también le permite al docente recuperar información acerca de sus principales fortalezas y debilidades, y con ello poder realizar ajustes para la mejora de los arreglos de las situaciones de enseñanza-aprendizaje que tienen lugar en el aula.

El aula (laboratorio o un escenario de práctica) es, sin duda, en donde tiene lugar el proceso instruccional en el que participan el docente, quien enseña y los estudiantes, quienes aprenden, así como los referentes de aprendizaje, que en conjunto constituyen un episodio interactivo en el que se estructuran diversas situaciones académicas. De acuerdo con Villalta y Martinic (2011), existe un amplio consenso tanto teórico como empírico acerca de la importancia que tienen las interacciones para auspiciar el conocimiento y el aprendizaje.

El tipo de interacciones que se establecen entre los diferentes actores del episodio instruccional, depende de ellos y de los componentes curriculares, ya que son en gran medida los que dirigen el tipo de interacción que ha de desarrollarse. Asimismo, los episodios instruccionales pueden darse o no, como una situación interactiva, ya que en algunas ocasiones se estructuran episodios pasivos en los que solo participa el docente o los estudiantes, sin que exista un intercambio recíproco.

Durante los últimos dos ciclos escolares en México (2017-2018, 2018-2019), la Secretaría de Educación Pública ha apostado por una metodología para construir 
mejores entornos de aprendizaje y con ello transitar a una educación de mayor calidad, mediante la utilización de videograbaciones de clases y su posterior análisis en cuerpos colegiados denominados Consejos Técnicos Escolares, en los que se pretende observar una clase muestra, seleccionada aleatoriamente con el objetivo de describir las diferentes competencias docentes y realizar comentarios y aproximaciones que realimentan las habilidades del docente con miras a conducir cambios en su práctica, o sí sus habilidades son las esperadas, conservar sus destrezas en el aula. Sin embargo, los análisis observacionales que se realizan en educación básica hasta el momento, carecen de herramientas como los sistemas observacionales, que parten de un enfoque teórico establecido y que permiten el análisis sistemático de los elementos que se pretenden observar, para así realizar mejoras en las prácticas docentes, cabe mencionar que actualmente el sistema de bachillerato en México se ha integrado a la educación obligatoria, y por lo tanto, es compatible con algunas prácticas derivadas desde educación básica.

Por ello, es relevante la realización de estudios que permitan dar cuenta de cómo se estructuran esas relaciones docente-estudiantes en el proceso de enseñanza-aprendizaje en entornos naturales, ya que de esta manera se brinda una aproximación metodológica con herramientas útiles y asequibles que permitan sistematizar los eventos a estudiar y que sirvan para auspiciar los episodios interactivos en las situaciones instruccionales en distintos niveles educativos.

La presente investigación, realizada en aulas de bachillerato en clases del área de ciencias, retoma los elementos de la metodología observacional para el estudio de las relaciones docente-estudiantes en situaciones didácticas, con base en los elementos teóricos de modelos interactivos que describen las habilidades del docente y del estudiante, expresadas como desempeño docente y desempeño del estudiante.

Desde esta perspectiva, la metodología observacional ha sido una herramienta útil en investigaciones en el entorno natural de educación, constituyendo una herramienta que puede contribuir a robustecer la investigación en los contextos educativos, para recabar información de los hechos que ocurren en situaciones cotidianas de enseñanza-aprendizaje. De acuerdo con Anguera (1983), la observación permite registrar la información tal como ocurre en los escenarios naturales, sin embargo, para realizar la recogida de datos observacionales es importante contar con fundamentos conceptuales y delimitación de categorías de análisis, así como de sistemas de registro de las observaciones, válidos y confiables. Por ello, es necesario contar con una aproximación teórica que permita establecer dimensiones e indicadores válidos armoniosos y correspondientes con el estudio de cierto hecho o fenómeno a estudiar.

La metodología observacional (MO) ha evolucionado considerablemente posicionando a las videograbaciones como una de las herramientas principales para el desarrollo de las investigaciones. Asimismo, el uso de la metodología 
observacional ha traído entre otros aportes, el desarrollo de protocolos de observación sistematizados en programas informáticos, que permiten ahorrar una gran cantidad de tiempo para el registro y análisis, y con altas propiedades de fiabilidad, que permiten su uso en otras condiciones similares. También, se han desarrollado diversos programas informáticos de registro, control y análisis de datos observacionales, como son los programas Hoisan, Mots, Lince y Theme Coder, entre otros (Anguera y Hernández-Mendo, 2014).

Desde la metodología observacional, se han abordado las interacciones didácticas en los salones de clase, desde diversas perspectivas. Por ejemplo, Candela (2001) refiere que un aporte generado desde la relación entre discurso y actividad, ha sido la distinción entre la participación social y las estructuras de organización del discurso en el aula y que entendido así, el discurso en el aula, permite realizar modificaciones en las acciones tanto del docente como del estudiante. Asimismo, con base en del modelo de análisis gramatical de la interacción lingüística en el aula, denominado IRRA (Edwards \& Mercer, 1988; Sinclair \& Coulthard, 1975), Mortimer y Machado (2002) analizaron vídeos de interacciones didácticas en clases de Química en secundarias de Brasil, de acuerdo con tres elementos de mediación: el género, el tema y el enfoque. El IRRA conformado por: I. Iniciación del diálogo por el profesor, R. Respuesta por el profesor, y RA. Realimentación por parte del profesor.

Por otra parte, en el contexto de la enseñanza en educación media superior y superior, se han reportado algunos estudios desde la metodología observacional, centrando la atención en las relaciones profesor -alumnos, con algunas variaciones en los instrumentos y las estrategias de recolección de información y de su análisis. Peralta y Roselli (2015), realizaron análisis de interacciones didácticas en el nivel universitario, en las carreras de Psicología y de Física, mediante una planilla de observación de los comportamientos, para describir las características de las clases y los tipos de interacciones. Con base en el análisis global cualitativo de las clases, encontraron tres tipos básicos de interacción: colaborativa, participativa guiada y expositiva. Los autores señalan, con base en sus resultados, que el tipo didáctico impuesto por el docente es variable.

Díaz, Borges, Valadez y Zambrano (2015), analizaron las buenas prácticas en el ámbito universitario en Tenerife (España) y en Guadalajara (México) mediante análisis secuencial, encontrando patrones significativos en: a) la retroalimentación de los profesores a las preguntas del alumnado, b) el refuerzo a la participación y c) el fomento a la participación de estudiantes. Asimismo, Borges y Falcón (2018), realizaron un estudio que consistió en el análisis de la función docente de Explicación, mediante el instrumento denominado PROFE (Protocolo de Observación de la Función de Explicación), con base en la grabación de ocho horas de docencia, lo que permitió registrar los comportamientos de los docentes durante una clase. Los principales hallazgos en esta investigación sobre 
el comportamiento del docente fueron los relativos a la frecuencia de algunas conductas durante el desarrollo de la clase, tales como, "explicación lineal" y "explicación con recursos".

De igual forma, Mares, Rivas, Rocha, Rueda y González (2018), investigaron con el uso de la metodología observacional en clases de Español y de Ciencias en diversos grados de la primaria mexicana, para ver si la implementación de la reforma educativa modificó patrones de interacción didáctica, comparando dos periodos escolares distantes, ciclo escolar 2002-2003 y ciclo escolar 20152016. Si bien es cierto que encontraron un incremento drástico en el uso de las tecnologías de la información y la comunicación y la participación de los alumnos durante las clases de Ciencias, no hubo incremento en las interacciones en niveles funcionalmente complejos, y que, en las clases del ciclo 2015-2016, solo en una tercera parte del tiempo de las clases, se presentaron interacciones para desarrollar las habilidades y competencias establecidas en la Reforma Educativa.

Al respecto, Bazán, Martínez y Trejo (2009), con base en la metodología observacional analizaron episodios interactivos que suceden en clases de primer grado de primaria en escuelas públicas de Sonora, México, en la asignatura de español. Los principales hallazgos sugieren que las interacciones de las clases observadas son mayoritariamente de menor complejidad funcional, pero que la enseñanza que propicia directamente experiencias y condiciones de mayores oportunidades de aprendizaje en niveles funcionalmente más complejos, marcan la diferencia tanto en el aprendizaje como en el logro académico, mostrando en ambos estudios que, el desempeño de los estudiantes se ajusta funcionalmente a los requerimientos y criterios del desempeño del profesorado en las diferentes temáticas de Español, de acuerdo con el currículo.

Además, la metodología observacional y los instrumentos de registro derivados, pueden ser utilizados para proporcionar a los docentes y directivos, oportunidades para la mejora de la práctica docente y de la calidad de la enseñanza (Jensen, Wallace, Steinberg, Gabriel, Dietiker, Davis \& Rui, 2019), así como para valorar aspectos de interculturalidad en las interacciones en situaciones de enseñanza (Jensen, Grajeda, \& Haertel, 2018).

Esta aproximación metodológica para estudiar un evento o fenómeno debe estar en correspondencia con un cuerpo teórico que ayude a sustentar el estudio y de esta misma forma se puedan hacer contribuciones a los modelos teóricos y empíricos. De este modo, los sistemas de categorías derivadas de supuestos teóricos sólidos, brindan la posibilidad de realizar un análisis sistemático, a partir de un enfoque o modelo teórico que le permite al investigador generar evidencia de los hechos específicos a estudiar. Tal como han señalado ya hace varias décadas Heyns \& Zander (1972), las categorías de análisis de observaciones que conforman a los sistemas de registro utilizados en MO, parten de teorías más desarrolladas, y 
de esta forma es posible hacer la identificación de una serie de eventos observados, de forma sistemática y organizada.

En este sentido, los instrumentos observacionales o también denominados sistemas de categorías, permiten establecer una estrategia para poder estudiar distintos fenómenos sistemáticamente, con el afán de ser más precisos en la aproximación a los diferentes constructos con referencia a un marco teórico específico.

\section{Criterios de desempeño en la interacción didáctica}

Para el presente estudio se recupera la noción de interacción didáctica. En el contexto de la enseñanza-aprendizaje, el docente despliega una serie de habilidades y las ejercita de acuerdo con ciertos criterios de efectividad, bajo una perspectiva teórica y ejemplar metodológico determinados, para desarrollar en sus estudiantes, comportamientos variados y efectivos. Este contacto interactivo corresponde al comportamiento del alumno mediado, quien, sí es que la relación de referencia es eficaz, aprenderá a comportarse de acuerdo con las contingencias y criterios de actuación y de logro, que el docente medio (Ibáñez y Ribes, 2001).

Este proceso interactivo se puede denominar interacción didáctica, y corresponde al ámbito de la didáctica general, en el que convergen la didáctica y la psicología aplicada en la educación (Ibáñez, 2014). Esta interacción didáctica implica el intercambio recíproco entre docente y estudiantes, y el objeto de aprendizaje o situaciones referentes, en condiciones definidas por el ámbito de desempeño-disciplina o profesión (Irigoyen, Acuña y Jiménez, 2011).

Retomando el modelo de Ribes (1993) sobre los juegos de lenguaje en la práctica científica, Carpio, Pacheco, Canales y Flores (1998), propusieron cinco ámbitos de desempeño en la práctica docente en psicología, los cuales podrían derivarse también a la práctica docente en otras disciplinas, dado que dichos ámbitos de desempeño son similares y relativamente constantes en términos del modo funcional de interacción que tienen lugar en la enseñanza, ya sea de la práctica científica, de la tecnológica o de la didáctica: 1) la exploración cognoscitiva o de marcos conceptuales del aprendiz, 2) la explicitación de criterios paradigmáticos y docentes, 3) la ilustración y ejemplificación, 4) la práctica en situaciones simuladas o reales y 5) la evaluación.

Más adelante, Irigoyen Acuña y Jiménez (2011), propusieron un modelo de interacción didáctica en el que destacan dos elementos principales. El primero se refiere a siete criterios de desempeño del docente: 1) planeación didáctica, 2) exploración competencial, 3) prescripción de criterios, 4) ilustración, 5) supervisión, 6) retroalimentación y 7) evaluación. el segundo elemento del modelo se refiere al desempeño del estudiante, el cual incluye cuatro criterios: 1) identificación de eventos, 2) instrumentación de procedimientos, 3) formulación de relaciones, y 4) aplicación de nuevos casos. 
Por otra parte, Silva, Morales, Pacheco, Camacho, Garduño y Carpio (2014), propusieron un modelo que puede ser utilizado para analizar el comportamiento de quien enseña en términos de diferentes habilidades que se utilizan para conseguir que alguien más aprenda: el Modelo Interconductual de Desempeño Didáctico (MIDD). Este modelo especifica siete criterios de desempeño docente: 1) planeación, 2) exploración competencial, 3) criterios, 4) ilustración, 5) supervisión de la práctica, 6) retroalimentación y 7) evaluación. De acuerdo con los autores, el MIDD no pretende dar cuenta del episodio interactivo de manera general, sino más bien, trata de hacerlo desde el punto del desempeño del docente, para poder auspiciar el aprendizaje de sus estudiantes.

Tomando como base las propuestas de los ámbitos o criterios de desempeño docente propuestos por Carpio et al. (1998), Irigoyen et al. (2011) y por Silva et al. (2014), en la tabla 1 se presenta un esquema que incluye cinco dimensiones o criterios de desempeño del docente y cinco dimensiones o criterios de desempeño del estudiante, que se relacionan entre sí, para dar cuenta de las interacciones didácticas que ocurren en un entorno natural de aprendizaje. En este esquema se incluye el término Ajuste, solo para caracterizar que durante un episodio interactivo, el estudiante satisface diferentes criterios de desempeño con base en cierta morfología y de acuerdo al nivel de aptitud funcional implicado, derivado de la práctica docente, y que se estructura en la medida en que se afecta la relación estudiantes-objeto referente de aprendizaje, mediada por el desempeño del docente y el discurso didáctico.

\section{Tabla 1}

Criterios de Desempeño Docente y Desempeño del Estudiante en la Interacción Didáctica

DOCENTE ESTUDIANTE

Exploración competencial Ajuste a la exploración competencial

Identifica los conocimientos previos de los estudiantes. Muestra precurrentes y potenciales habilidades.

\begin{tabular}{ll}
\hline Explicitación de criterios & Identificación de criterios \\
Explica los parámetros que el estudiante deberá & Reproduce y/o recrea los criterios del curso o de la \\
satisfacer de acuerdo con los criterios didácticos. & $\begin{array}{l}\text { clase; pregunta sobre qué y cómo se debe ajustar a los } \\
\text { criterios. }\end{array}$
\end{tabular}

Ilustración
Muestra y relaciona al estudiante con el actuar efectivo
para el cumplimiento de algún criterio mediante las
acciones propias, y las del estudiante.

\section{Realimentación}

Pone en contacto al estudiante con su desempeño, $\mathrm{y}$ muestra las formas en las que puede satisfacer el criterio.

\section{Ajuste al modo lingüístico y a la ilustración \\ Actúa según los criterios designados por el docente en función de los requisitos didácticos, y según el modo lingüístico pertinente.}

Ajuste del alumno a la realimentación

Se pone en contacto con su propia ejecución y permite realizar cambios en su actividad respecto a los criterios y observaciones realizadas por el docente o por él mismo.

\footnotetext{
Evaluación

Contrasta el desempeño del estudiante con el desempeño programado.
}

Participación y aplicación
Realiza las actividades, ejercicios, resuelve problemas,
implementa procedimientos en función de logro,
efectividad y variabilidad.

Fuente: Elaboración propia. 
La presente propuesta considera la correspondencia entre ámbitos o criterios para el docente y para el estudiante, respecto a su número y concordancia teórica, con la finalidad de contar con un sistema que permita analizar las interacciones didácticas que ocurren en entornos formales de educación.

\section{Objetivos de la investigación}

Se diseñó el presente estudio con el objetivo de construir un sistema observacional con cinco ámbitos de desempeño del docente y del estudiante así como, validarlo para realizar análisis de interacciones didácticas en clases de bachillerato, bajo el enfoque interconductual de la psicología. Un segundo objetivo fue describir de forma exploratoria, las relaciones entre criterios de desempeño del docente y criterios de desempeño del estudiante, en dos clases de ciencias (Matemáticas y Biología) en un bachillerato de una universidad pública en el Estado de Morelos, México.

\section{Método}

\section{Diseño}

El presente estudio se cataloga como; ideográfico, puntual y multidimensional (I/P/M), catalogado así por sus características. En cuanto a su unidad de estudio se denomina ideográfico ya que los participantes se integran en una única unidad, en cuanto a su temporalidad se considera puntual ya que ocurre en un momento determinado sin que interese el proceso o bien, las sesiones sucesivas, y por último, se considera multidimensional ya que existen una heterogeneidad de posibilidades de catalogar las interacciones, de acuerdo con los diseños observacionales (Anguera, Blanco, Hernández, y Losada, 2011).

\section{Participantes}

La selección de los participantes se realizó mediante un muestro no probabilístico intencional (Hernández, Fernández, y Baptista, 2010). Se seleccionaron dos docentes y sus grupos de estudiantes de clases en el área de ciencias en el segundo semestre de Bachillerato (educación media superior) de una escuela pública en el Estado de Morelos, México. El primer grupo, conformado por 35 estudiantes con edades de 16 a 17 años, de la asignatura de matemáticas II, cuyo docente es varón. El segundo grupo de 37 estudiantes con edades de 16 a 17 años, de la asignatura de biología II, cuyo docente es mujer.

\section{Instrumento}

Se diseñó el Sistema Observacional Para Analizar Interacciones Didácticas (SOPAID), que contiene cinco dimensiones para el docente y cinco dimensiones para el estudiante y al menos tres indicadores válidos para cada una. Desempeño docente: 1) exploración competencial, 2) explicitación de criterios, 3) ilustración, 4) 
realimentación y 5) evaluación. para el desempeño del estudiante las dimensiones fueron: 1) ajuste a la exploración competencial, 2) identificación de criterios, 3) ajuste al modo lingüístico y a la ilustración 4) ajuste del alumno a la realimentación, 5) participación y aplicación (ver Apéndice 1).

\section{Aparatos}

Cámara para videograbar marca SONY Handycam HDR-PJ230, un teléfono marca Motorola G4 play con videocámara, un tripie marca vivitar y el sotfware Lince versión 1.4 para realizar el análisis de los materiales de video.

\section{Materiales}

- $\quad$ Software LINCE versión 1.4

- Hoja de categorías del-Sistema Observacional Para el Análisis de Interacciones Didácticas (SOPAID)

Aspectos éticos

Durante el inicio, desarrollo y conclusión del estudio se llevó a cabo un protocolo de actuación de acuerdo con aspectos éticos, en primer lugar se pidió permiso institucional mediante un oficio en el que se explicaba todos los alcances y posibles beneficios del proyecto a la institución, posteriormente se platicó con los profesores participantes para pedirles su aprobación para poder ser videograbados; antes de eso se les explicaron los alcances y posibles beneficios del proyecto a su práctica profesional, asimismo, antes de iniciar las grabaciones en las clases hubo una sesión en la que se les explicó a los estudiantes el proyecto y se les mencionó cuales eran los derechos de los estudiantes a querer participar o no, en el estudio, por último se pidieron a todos los participantes que firmaran un formato de consentimiento y asentimiento informado respectivamente.

\section{Procedimiento}

La primera fase del estudio consistió en la generación de las categorías de observación que describen las interacciones didácticas, tomando en cuenta el desempeño del docente y el desempeño del estudiante como un intercambio recíproco entre los individuos que participan en la interacción, de acuerdo con algunos componentes de los modelos de interacción didáctica de (Carpio, et al. 1998; Irigoyen et al. 2011; Silva et al. 2015).

La segunda fase consistió en realizar dos videograbaciones de clases de 50 minutos por módulo, una en la asignatura de Matemáticas II y otra en la asignatura Biología II, en el tercer bloque de estudio correspondiente al segundo semestre de bachillerato, en el mes de octubre, en el semestre regular agostodiciembre de 2017. 
En la tercera fase se hizo la programación de las categorías de observación en el software LINCE para su utilización en el análisis de las interacciones didácticas, se probaron las categorías observacionales en los dos videos de las filmaciones y posteriormente se realizaron los análisis de las interacciones de enseñanza-aprendizaje con base en las categorías. Asimismo, se realizaron análisis de fiabilidad para saber si es que existía un grado aceptable de concordancia entre los registros de dos observadores a quienes se les capacitó durante dos meses. En la prueba para la confiabilidad entre registradores, a cada registrador se les dio la siguiente instrucción: "Con base en el sistema observacional, registrarás todos los elementos que caracterizan una interacción de un módulo de clase de matemáticas y biología respectivamente", después de que concluyeron los registros independientes, se procedió a realizar el análisis de concordancia entre los dos registros que hicieron por cada clase.

Programación del sistema observacional en el software y determinación del acuerdo interobservadores

Respecto al sistema observacional para analizar el desempeño del docente y de los estudiantes de acuerdo con cinco dimensiones cada por cada uno, en la figura 1 se muestra la programación de las dimensiones y los indicadores en el software Lince 1.4. El sistema de registro mostrado incluye un rótulo general del campo Interacciones Didácticas, categorías de datos generales y las variables que se refieren a criterios de inicio del segmento interconductual, a criterios de desempeño del docente y a criterios de desempeño del estudiante.

Posterior a la programación, se planteó validar el sistema observacional, para lo cual se inició con el registro de cada clase. En la Figura 2 se presenta un registro desde el software Lince 1.4, que ejemplifica como se llevó a cabo uno de los registros de clase (biología), en el que se muestra la videograbación de un módulo de 50 minutos en el cual la profesora desarrolla el tema de metabolismo y fotosíntesis. En el lado inferior izquierdo de la figura $2 \mathrm{se}$ puede observar el registro de la clase, con el tiempo en el que ocurre un episodio interactivo, quién los inicia, así como las diferentes secuencias registradas.

\section{Análisis estadísticos}

Para el análisis de Fiabilidad del sistema observacional, se utilizó un coeficiente Kappa de Cohen para determinar el acuerdo inter-observadores, en el que mientras más cercano a +1 , tendrá un mayor acuerdo entre los observadores, y entre más cercano sea a -1 , tendrá un menor grado de acuerdo entre los observadores de acuerdo con Cerda y Villarroel (2008). 
Para la descripción del desempeño del docente y el desempeño de los estudiantes como una interacción didáctica, se realizó estadística descriptiva, con el análisis de frecuencia de los criterios registrados del docente y de los estudiantes.

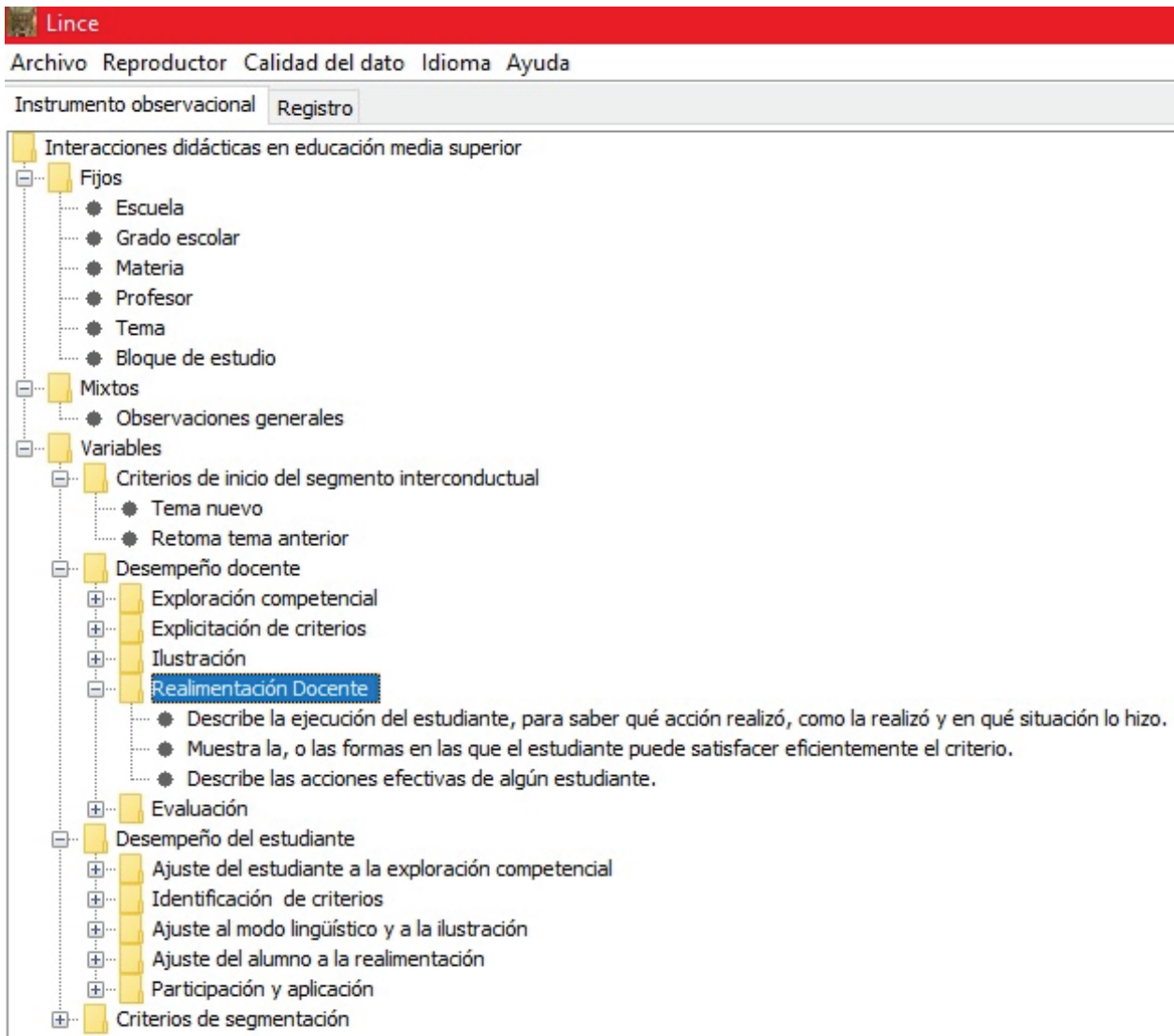

†.. Criterios de segmentación

Figura 1. Programación de las dimensiones del sistema observacional para analizar interacciones didácticas en Bachillerato, en el software Lince 1.4. 


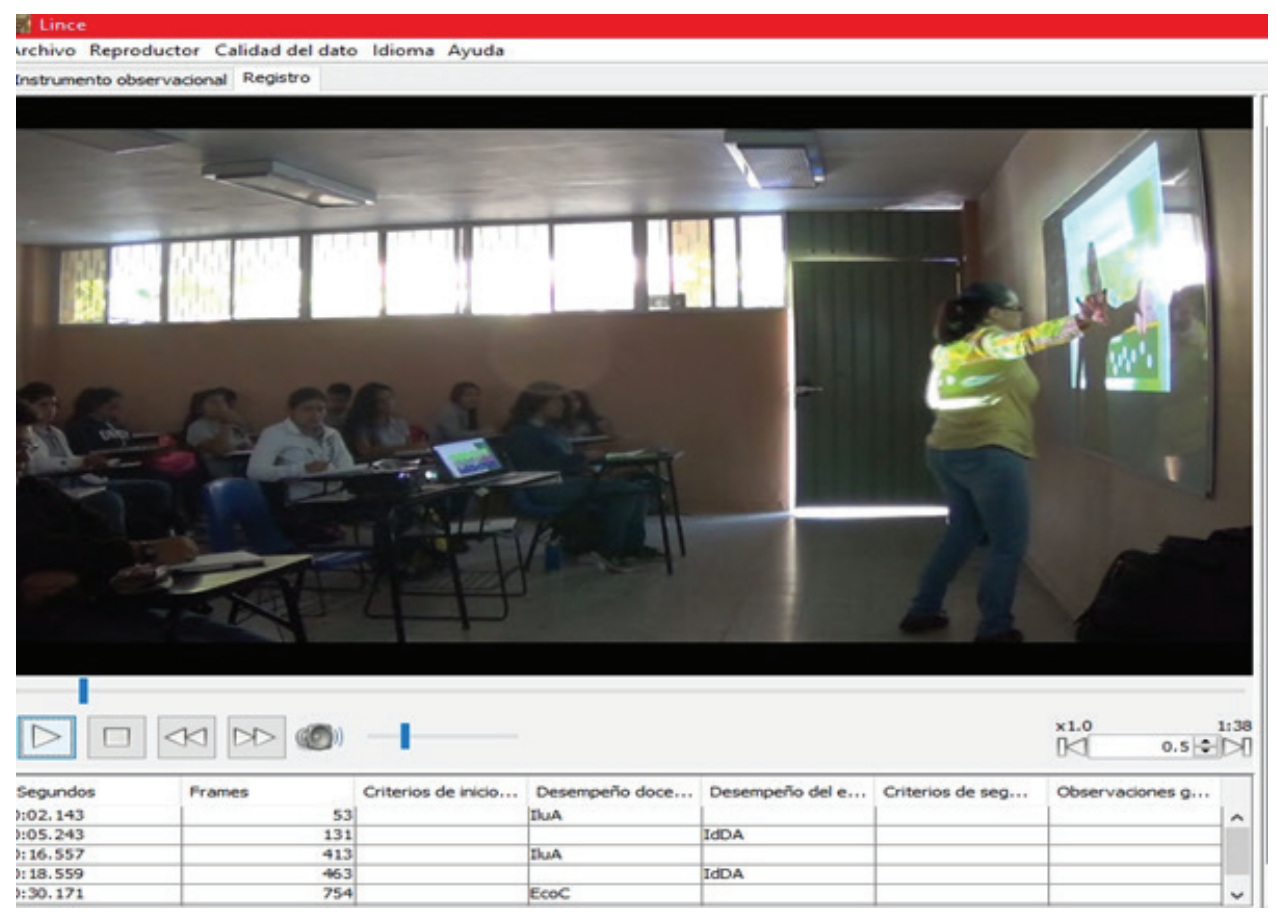

Figura 2. Registro de videograbación de una clase de Biología con el software Lince versión 1.4.

\section{RESULTADOS}

Para el análisis de concordancia entre registradores, para el primer registro se obtuvo un coeficiente Kappa $(\mathrm{K})=.822$, lo cual indica que el grado de acuerdo entre los observadores es el denominado considerable, de acuerdo Landis y Koch (1977). Para el segundo registro el puntaje $K=.798$, es decir que si bien bajó respecto al primer registro, el grado de acuerdo entre los observadores se mantiene como un grado de acuerdo denominado considerable, tal como se presenta en la tabla 2.

\section{Tabla 2}

Determinación del acuerdo entre observadores

\begin{tabular}{lcc}
\hline & Valor en el primer registro & Valor en el segundo registro \\
\cline { 2 - 3 } Medida de acuerdo (Kappa) & .822 & .798 \\
\hline $\mathrm{N}$ de casos válidos & 44 & 44 \\
\hline
\end{tabular}

Nota: Índice Kappa=.822 y P $>0.05$ para el primer registro e Índice Kappa=.798 y P $>0.05$ para el segundo registro que de acuerdo con los parámetros del índice de concordancia se encuentra en un nivel de estimación denominada considerable.

En cuanto a las relaciones entre criterios de desempeño del docente y del alumnado, en la figura 3 se muestra el análisis de interacciones en la clase de 
matemáticas, en la cual se muestra que, los criterios que se registraron con mayor frecuencia fueron Ilustración por parte del docente y Ajuste al modo lingüístico y a la Ilustración por parte del alumnado. La figura 3 también muestra que, los criterios menos registrados fueron: Realimentación del docente y el ajuste del alumno a la realimentación. Dos criterios de desempeño no se presentaron en esta clase: Explicitación de criterios y Evaluación, debido a que la naturaleza de la clase se tornó en función de la explicación del tema de la hipérbola, sin embargo, no se requirió realizar algún ejercicio práctico en el que las competencias del docente fueran distintas a la prevalencia de su discurso en el desarrollo de la clase.

\section{CLASE DE MATEMÁTICAS}

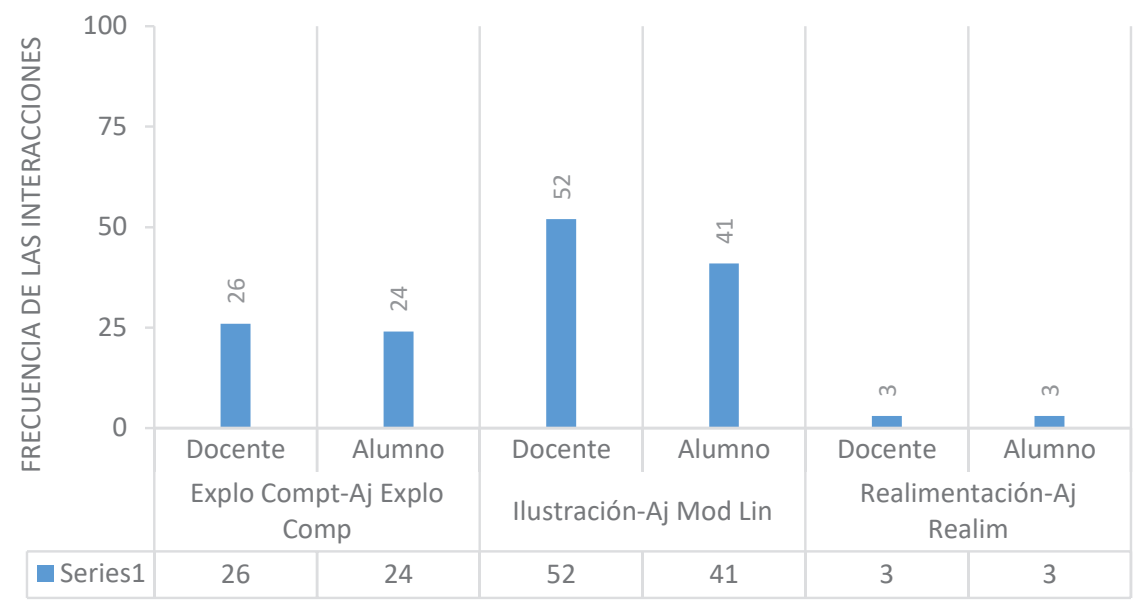

Figura 3. Criterios de desempeño y del estudiante en clase de matemáticas

En el análisis del registro de la segunda clase (Biología), datos similares a la primera clase, ya que nuevamente son tres criterios de los cinco posibles los que se registran, en este caso: Exploración competencial - Ajuste del alumno a la exploración competencial, Realimentación - Ajuste a la realimentación e Ilustración - Ajuste al modo lingüístico y a la Ilustración (ver figura 2). La interacción de los criterios de desempeño Ilustruación fue la que se presentó con mayor frecuencia 


\section{CLASE DE BIOLOGÍA}

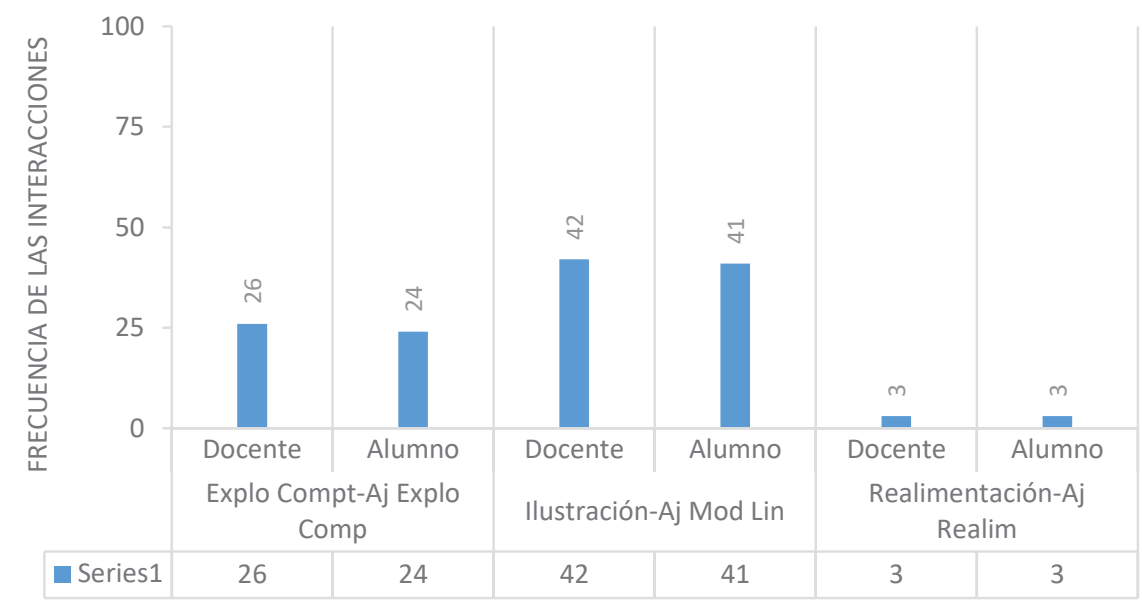

Figura 4. Segmento interactivo en clase de Biología

\section{DISCUSIÓN}

El primer objetivo de esta investigación fue construir y validar un sistema observacional para analizar el desempeño del docente y de los estudiantes de acuerdo con cinco dimensiones de interacción didáctica. De acuerdo con los resultados obtenidos, el instrumento observacional utilizado en este estudio, sí responde a la necesidad de que los instrumentos observacionales cumplan con criterios de fiabilidad, como parámetros fundamentales de la metodología observacional y que sirvan para hacer análisis desde diferentes áreas de conocimiento (Bakeman \& Gottman, 1989; Anguera, 1983).

En este caso, el instrumento observacional realizado para analizar las interacciones didácticas, cumple con los criterios de fiabilidad (primer registro $K=822$, segundo registro $K=.798$ ), para hacer aproximaciones en entornos naturales de educación media superior; índices que coinciden con los parámetros de fiabilidad reportados por Borges y Falcón (2018), quienes sometieron su sistema observacional para el acuerdo entre registradores de interacciones en clases a nivel universitario, obteniendo puntajes de $K=.622$ en la primer versión del instrumento y $K=.866$ en su segunda versión.

Los resultados de este estudio con el Sistema Observacional Para el Análisis de Interacciones Didácticas (SOPAID), coinciden con los hallazgos reportados en otros estudios que derivan sistemas observacionales para analizar eventos relacionados con procesos de interacciones docente - estudiantado en la enseñanza-aprendizaje (Díaz, Borges, Valadez y Zambrano, 2015; Borges y Falcón 
2018; Fabra, Balaguer, Tomás, Smith y Duda, 2018), que en conjunto proporcionan evidencian de la importancia de validar los sistemas observacionales con los que se pretende realizar un trabajo de investigación. De esta forma, mediante el acuerdo entre registradores, se asegura la posibilidad de que la información recabada no sea única e irrepetible, adecuándose a parámetros de fiabilidad.

Los resultados de la construcción y validación del sistema observacional que aquí se reportan, también permiten valorar la pertinencia de los sistemas observacionales con base en una teoría sustantiva que enmarca tanto las categorías derivadas, como los criterios e indicadores de tales categorías, en los sistemas de registro. En el presente trabajo, tomando cinco criterios de desempeño del docente en la práctica didáctica a partir de los modelos teóricos de interacción didáctica propuestos por Carpio et al. (1998), Irigoyen et al. (2011) y por Silva et al. (2014), concretamos cinco dimensiones o ámbitos de desempeño para el docente y para el estudiante, es decir, concretamos desempeños tato para los docentes como para los estudiantes, en cada uno de los cinco criterios o ámbitos de desempeño en interacciones didácticas.

Respecto al segundo objetivo de este trabajo, referente a la exploración de las situaciones de interacción en dos clases de ciencias (matemáticas y biología), se encontró que tres de las cinco categorías posibles fueron registradas mayoritariamente, una posible explicación es que los profesores agrupan su actividad docente en la actividad más común dentro del aula, la cual es explicar el tema sin fomentar la interacción entre compañeros o docente compañeros.

De acuerdo con Villalta, Assael y Martinic (2013), la posibilidad de contar con herramientas que permitan describir la organización de los procesos de enseñanza y aprendizaje nos pueden permitir orientar la intervención del docente hacía un mejor logro en su papel como mediador del aprendizaje. En este mismo sentido, las investigaciones que se han realizado en educación básica (Guevara, Mares, Rueda, Rivas, Sánchez y Rocha, 2004; 2005; Bazán, Martínez y Trejo 2009), permiten conocer, no solo el tipo de interacciones que se establecen respecto al tipo de asignatura, sino también permiten estudiar el nivel de dificultad en el que están planteados los aprendizajes de los estudiantes.

Una limitación aparente de este estudio es el número de observaciones, pero que es permitido en $\mathrm{MO}$ dado que la observación de una sola clase entra como parte del diseño ideográfico y puntual, que hemos mencionado en la sección Método, pero cabe resaltar que si bien es cierto, dado el tamaños de la muestra de las observaciones, la validación de esta pertinencia tendría que tener un fuerte énfasis en el análisis lógico conceptual de las categorías derivadas de una teoría o de modelos derivados de una teoría sobre el desarrollo del comportamiento y sobre las propias interacciones didácticas, los índices de fiabilidad entre observadores 
puede estar indicando la exhaustividad y mutua exclusión tanto de los indicadores, así como de las categorías de los ámbitos de desempeño del docente y del estudiante.

Futuros estudios tanto con análisis secuencial de interacciones y mayor número de observaciones por clase, así como con el uso de cuestionarios o escalas derivadas de estas categorías, permitirán una valoración más amplia y complementaria a los registros observacionales puntuales, sobre lo que puede estar ocurriendo en las clases, de acuerdo con los ámbitos de desempeño didáctico.

\section{CONCLUSIONES}

1. Existe un grado de acuerdo entre observadores denominado "considerable" para la presente investigación, lo que sugiere la posibilidad del uso de esta herramienta en otros contextos y niveles educativos.

2. Las principales categorías que se registraron en el estudio exploratorio muestran que los docentes realizan mayoritariamente actividades de explicación y cuestionamientos acerca de lo que saben antes de iniciar con un tema, de esta forma, las categorías de las cuales se prescindieron en estas aproximaciones fueron: explicitación de criterios y evaluación.

3. Existe la posibilidad de variar las situaciones de enseñanza-aprendizaje para poder incidir en la dinámica escolar mediante la creación de arreglos para sugerir otro tipo de interacción en el aula.

\section{REFERENCIAS}

Anguera, M. T (1983). Manual de prácticas de observación. México: Trillas.

Anguera, M., Blanco, A., Hernández, A. y Losada J. (2011). Diseños observacionales: ajuste y aplicación en psicología del deporte. Cuadernos de psicología del deporte, 11(2), 63-76.

Anguera, M., T. y Hernández-Mendo, A. (2014). Metodología observacional y psicología del deporte: Estado de la cuestión. Revista de psicología del deporte, 23(1), 103-109.

Bakeman, R. \& Gottman, J. (1989). Observación de la interacción: Introducción al análisis secuencial. Madrid: Ediciones Morata, S. A.

Bazán, A., Martínez, X. y Trejo, M. (2009). Análisis de Interacciones en Clases de Español de Primer Grado de Primaria. Revista Interamericana de Psicología, 43(3), 466-478.

Borges, M. A. y Falcón, C. (2018). Protocolo de observación de la función de explicación (PROFE): un instrumento para operacionalizar la transmisión de contenidos por parte del profesorado. Universitas Psychologica, 17(3), 1-12.

Candela, A. (2001). Corrientes teóricas sobre discurso en el aula. Revista Mexicana de Investigación Educativa, 6, 317-333. 
Carpio, C., Pacheco, V., Canales, C. y Flores, C. (1998). Comportamiento inteligente y juegos de lenguaje en la enseñanza de la psicología. Acta comportamentalia: Revista latina de análisis del comportamiento, 6(1), 47-60.

Díaz, M. C., Borges, A. Valadez, M. D. y Zambrano, R. (2015). Valoración de buenas prácticas docentes a través de observación sistemática. Universitas Psychologica, 14(3), 913-922.

Edwards, D., \& Mercer, N. (1988). El conocimiento compartido: el desarrollo de la comprensión en el aula. Barcelona, España: Paidós

Fabra, P., Balaguer, I., Tomás, I., Smith, N., y Duda, J., (2018). Versión española del clima motivacional multidimensional (MMCOS): fiabilidad y evidencias de validez. Revista de psicología del deporte, 27(1), 11-22.

Guevara, Y., Mares, G., Rueda, E., Rivas, O., Sánchez, B. y Rocha, E. (2004). Niveles de interacción que se propician en alumnos de educación primaria durante la enseñanza de la materia español. Revista Mexicana de Análisis de la conducta, 31(1), 22-45.

Guevara, Y., Mares, G., Rueda, E., Rivas, O., Sánchez, B. y Rocha, E. (2005). Análisis de las interacciones maestra-alumnos durante la enseñanza de las ciencias naturales en primaria. Revista Mexicana de Investigación Educativa, 9(22) 721-745.

Heyns, R. y Zander, A. (1972). Observación de la conducta de grupo. En Festinger, L. y Katz, D. (eds.). Los métodos de investigación en las ciencias sociales. pp. $251-289$. Buenos Aires: Paidós.

Hernández, R., Fernández, C., y Baptista, P. (2010). Metodología de la investigación. México: Mc Graw Hill Educación.

Ibañez C. y Ribes, E. (2001). Un análisis interconductual de los procesos educativos. Revista Mexicana de Psicología, 18(3), 359-371.

Ibáñez, C. (2014). Problemas de la psicología aplicada a la educación. En A. Bazán y D. Castellanos (Coordinadores), La psicología en la educación: Contextos de aprendizaje e investigación (pp. 11-31). México: UAEM - Plaza y Valdés.

Irigoyen, J, Acuña, F. y Jiménez (2011). Interacciones didácticas en educación superior. Algunas consideraciones sobre la evaluación de desempeños. En J. Irigoyen, F. Acuña, y M. Jiménez (Coordinadores), Evaluación de desempeños académicos (pp. 73-96). Hermosillo: Interactum.

Jensen, B., Wallace, T. L., Steinberg, M. P., Gabriel, R. E., Dietiker, L., Davis, D. S., Rui, N. (2019). Complexity and scale in teaching effectiveness research: Reflections from the MET Study. Education Policy Analysis Archives, 27(7). http://dx.doi. org/10.14507/epaa.27.3923

Jensen, B., Grajeda, S., \& Haertel, E. (2018). Measuring cultural dimensions of classroom interactions. Educational Assessment, 23(4), 250-276. https://doi.org/10.1080/106271 97.2018.1515010

Landis J, Koch G. (1977). The measurement of observer agreement for categorical data. Biometrics, 33(1), 159-74. 
Mares, G., Rivas, O., Rocha, H., Rueda, E., \& González, L. F. (2018). Exploración del efecto de la Reforma Integral de la Educación Básica sobre las interacciones en el aula. Revista mexicana de investigación educativa, 23(77), 547-575.

Mortimer, E., \& Machado, A. (2002). Mecanismos de mediación, flujo del discurso y el significado en clase de ciencias. En M. Benlloch (Ed.), La educación en ciencias: ideas para mejorar su práctica (pp. 217-232). Barcelona: Paidós.

Peralta, N. S., \& Roselli, N. D. (2015). Los sistemas de interacción generados por la impronta didáctica del docente. Propósitos y representaciones, 3(2), 131-177.

Ribes, E. (1993). La práctica de la investigación científica y la noción de juego de lenguaje. Acta Comportamentalia. 1(1), 63-82.

Silva, H., Morales, G., Pacheco, V., Camacho, A., Garduño H. y Carpio C. (2014). Didáctica como conducta: una propuesta para la descripción de las habilidades de enseñanza. Revista Mexicana de Análisis de la Conducta, 40(3), 32-46.

Sinclair, J., \& Coulthard, M. (1975). Towards an analysis of discourse. The English used teachers and pupils. London: Oxford University Press.

Villalta, P., Assael, M. y Martinic, S., (2013). Conocimiento escolar y procesos cognitivos en la interacción didáctica en la sala de clase, Perfiles Educativos, 35(141), 84-96. 
Apéndice 1: Sistema Observacional Para el Análisis de Interacciones Didácticas (SOPAID)

\begin{tabular}{|c|c|c|c|}
\hline Criterio & Descripción & Código & Indicadores \\
\hline \multicolumn{4}{|c|}{ Criterios para el desempeño docente } \\
\hline $\begin{array}{l}\text { Exploración } \\
\text { competencial }\end{array}$ & $\begin{array}{l}\text { Identifica los conocimientos } \\
\text { previos de los estudiantes. }\end{array}$ & ECo & $\begin{array}{l}\text { A. Realiza preguntas a los estudiantes antes de } \\
\text { iniciar un tema. } \\
\text { B. Plantea problemas a los estudiantes para que los } \\
\text { resuelvan antes de iniciar un tema. } \\
\text { C. Pregunta conceptos referentes al tema antes de } \\
\text { explicarlos }\end{array}$ \\
\hline $\begin{array}{l}\text { Explicitación de } \\
\text { criterios }\end{array}$ & $\begin{array}{l}\text { Explica los parámetros que el } \\
\text { estudiante deberá satisfacer } \\
\text { de acuerdo con los criterios } \\
\text { didácticos. }\end{array}$ & $\mathrm{ECr}$ & $\begin{array}{l}\text { A. Describe los requerimientos necesarios para } \\
\text { realizar algún trabajo en clase. } \\
\text { B. Describe las acciones que el estudiante deberá } \\
\text { cumplir para realizar una práctica. } \\
\text { C. Explica lo que el estudiante debe hacer para } \\
\text { completar un ejercicio en clase. }\end{array}$ \\
\hline Ilustración & $\begin{array}{l}\text { Muestra y relaciona al } \\
\text { estudiante con el actuar } \\
\text { efectivo para el cumplimiento } \\
\text { de algún criterio mediante } \\
\text { las acciones propias, y las del } \\
\text { estudiante. }\end{array}$ & ILu & $\begin{array}{l}\text { A. Explica el tema de la clase. } \\
\text { B. Resuelve problemas frente al estudiante. } \\
\text { C. Ejemplifica la tarea que asigna al estudiante. } \\
\text { D. Modela las acciones específicas para cumplir con } \\
\text { la práctica de la clase. }\end{array}$ \\
\hline Realimentación & $\begin{array}{l}\text { Pone en contacto al } \\
\text { estudiante con su desempeño, } \\
\text { y muestra las formas en } \\
\text { las que puede satisfacer el } \\
\text { criterio. }\end{array}$ & RtrD & $\begin{array}{l}\text { A. Describe la ejecución del estudiante, para saber } \\
\text { qué acción realizó, como la realizó y en qué situación } \\
\text { lo hizo. } \\
\text { B. Muestra la, o las formas en las que el estudiante } \\
\text { puede satisfacer efectivamente el criterio. } \\
\text { C. Describe las acciones efectivas de algún } \\
\text { estudiante. }\end{array}$ \\
\hline
\end{tabular}

\begin{tabular}{ll}
\hline Evaluación & $\begin{array}{l}\text { Contrasta el desempeño del } \\
\text { estudiante con el desempeño } \\
\text { programado }\end{array}$
\end{tabular}

A. Verifica si el estudiante contesta

equivocadamente.

B. Verifica si el estudiante contesta parcialmente.

C. Verifica si el estudiante contesta correctamente.

\section{Criterios del desempeño del estudiante}

\begin{tabular}{|c|c|c|c|}
\hline $\begin{array}{l}\text { Ajuste del } \\
\text { estudiante a la } \\
\text { Exploración } \\
\text { competencial }\end{array}$ & $\begin{array}{l}\text { Muestra precurrentes y } \\
\text { potenciales habilidades. }\end{array}$ & EcoE & $\begin{array}{l}\text { A. Responde al docente cuando le pregunta antes de } \\
\text { iniciar un tema. } \\
\text { B. Resuelve los problemas que el docente plantea } \\
\text { antes de iniciar un tema. } \\
\text { C. Responde las preguntas que el profesor hace } \\
\text { acerca de un tema nuevo. }\end{array}$ \\
\hline $\begin{array}{l}\text { Identificación de } \\
\text { criterios }\end{array}$ & $\begin{array}{l}\text { Reproduce y/o recrea los } \\
\text { criterios del curso o de la } \\
\text { clase; pregunta sobre qué y } \\
\text { cómo se debe ajustar a los } \\
\text { criterios. }\end{array}$ & $\mathrm{CmC}$ & $\begin{array}{l}\text { A. Cumple con los criterios que el profesor indica } \\
\text { para realizar algún trabajo en clase. } \\
\text { B. Realiza una práctica cumpliendo con los criterios } \\
\text { que el docente explicó. } \\
\text { C. Cumple con los criterios que el profesor menciona } \\
\text { al realizar algún ejercicio en clase. }\end{array}$ \\
\hline $\begin{array}{l}\text { Ajuste al modo } \\
\text { lingüístico y a la } \\
\text { ilustración }\end{array}$ & $\begin{array}{l}\text { Actúa según los criterios } \\
\text { designados por el docente } \\
\text { en función de los requisitos } \\
\text { didácticos, y según el modo } \\
\text { lingüístico pertinente. }\end{array}$ & IdD & $\begin{array}{l}\text { A. Se orienta hacía el profesor cuando explica el } \\
\text { tema. } \\
\text { B. Repite las frases que el docente le solicita. } \\
\text { C. Los estudiantes toman apuntes del tema. } \\
\text { D. Pregunta si algo no le queda claro acerca de la } \\
\text { explicación del tema. }\end{array}$ \\
\hline $\begin{array}{l}\text { Ajuste del } \\
\text { alumno a la } \\
\text { realimentación }\end{array}$ & $\begin{array}{l}\text { Se pone en contacto con } \\
\text { su actuar y modifica su } \\
\text { ejecución con base en la } \\
\text { supervisión del docente. }\end{array}$ & RtrA & $\begin{array}{l}\text { A. Refiere su propio actuar, identificado por el } \\
\text { docente o por él mismo y modifica su actuar. } \\
\text { B. Cambia sus acciones para cumplir con los } \\
\text { requisitos de la tarea. } \\
\text { C. Refiere las acciones que le faltaron para completar } \\
\text { la actividad correctamente. }\end{array}$ \\
\hline
\end{tabular}




\begin{tabular}{|c|c|c|c|}
\hline Criterio & Descripción & Código & Indicadores \\
\hline \multicolumn{4}{|c|}{ Criterios del desempeño del estudiante } \\
\hline $\begin{array}{l}\text { Participación } \\
\text { y aplicación }\end{array}$ & $\begin{array}{l}\text { Interviene en la clase para } \\
\text { emitir su opinión acerca } \\
\text { del tema o realizar algún } \\
\text { ejercicio o actividad }\end{array}$ & Par & $\begin{array}{l}\text { A. Complementa el comentario del profesor o de sus } \\
\text { compañeros. } \\
\text { B. Emite su opinión acerca de un tema. } \\
\text { C. Pregunta al profesor acerca del tema que se está } \\
\text { desarrollando. } \\
\text { D. Realiza una explicación acerca del tema que se } \\
\text { está desarrollando. } \\
\text { E. Realiza alguna actividad de acuerdo con el tema. } \\
\text { F. Resuelve algún problema planteado por el docente. } \\
\text { G. Implementa algún procedimiento de acuerdo con } \\
\text { el tema de la clase. }\end{array}$ \\
\hline
\end{tabular}

